\title{
Introducing a New Type of Pathologic Tooth Migration In A Patient with Periodontal Disease
}

Mortazav H, Rezaeifar K, Anbari F* and Rahmani MS

Department of Oral Medicine, Dental School, Shahid Beheshti University of Medical Sciences, Tehran, Iran

\begin{abstract}
Pathologic Tooth Migration (PTM) is a common complication of moderate to severe periodontitis. Etiology of PTM appears to be multifactorial. Forces from surrounding soft tissues are known to be a cause. In addition, a wide variety of conditions have been suggested to be in association with PTM, including periodontal and periapical inflammation, bone loss, occlusal interferences, traumatic occlusion, class II malocclusion, shortened dental arch, missing teeth, etc. This condition is classified into five subgroups 1-facial flaring 2-diastema 3-extrusion 4-rotation 5-proximal tilting/tipping/ or drifting. Hereby we present an unusual case of PTM and suggest a modification in the current classification of this pathology.
\end{abstract}

Keywords: Pathologic tooth migration; Avulsion; Extrusion; Facial flaring; Diastema; Rotation; Tilting

\section{Introduction}

The position of the tooth in the dental arch depends on the healthiness and height of the periodontal compartments. It is also dependent on the forces exerted on the tooth, mainly from occlusion, lips, tongue, and cheeks [1]. Migration of a tooth is defined as the tooth movement from its primary position to another position in relation to the basal bone of the alveolar process, the opposing and the adjacent teeth [2]. Furthermore, tooth displacement may happen as a result of periodontal diseases that potentially can disturb the balances among the factors which maintain normal tooth position. This has been called Pathologic Tooth Migration (PTM) (1). The prevalence of PTM is ranged from $30.03 \%$ to $55.8 \%$ in patients with moderate to severe periodontitis [3]. The main etiology of PTM is not clear, however, a wide variety of conditions have been suggested to be in association with this phenomenon, including periodontal and periapical inflammation, bone loss, occlusal interferences, traumatic occlusion, class II malocclusion, habits (lip biting, tongue thrusting, pipe smoking, playing of wind instruments, bruxism, grinding, protrusive pattern of mastication and mouth breathing), soft tissue pressure, position of the tongue, shortened dental arch, missing teeth, extended lip frenula, and gingival enlargement due to pharmacotherapy [4-7]. More than one of these conditions may be present in a patient with PTM and a single tooth or a group of teeth can be affected [1,3]. In periodontal cases, the inflammatory destruction of the periodontium and pressure from the granulomatous tissue of the infra-bony periodontal pocket, bring about an imbalance between maintenance and migratory forces of the tooth [4]. PTM usually occurs in the anterior regions of the mouth but the involvement of posterior teeth is not uncommon [2]. The aim of this case report is to present an extraordinary type of PTM which up to our knowledge has not been reported yet in a patient with periodontitis.

\section{Case Report}

A 56-year old man with a chief complaint of tooth loss, tooth mobility and problems in biting and chewing attended oral medicine department of dental school of Shahid Beheshti University of medical sciences, Tehran, Iran. The patient was a smoker and had a history of hypertension; mitral valve prolapse and sleep apnea. He also used some medications including losartan, hydrochlorothiazide, aspirin, and amlodipine. The intraoral evaluation revealed a poor oral hygiene and posterior tooth loss. There was bleeding on probing. A pathologic migration with an unusual position of the right mandibular second molar was detected (Figure 1). The patient had a panoramic radiograph which has been taken about two years ago. There were periapical lesions and periodontal pockets in both arches. The migrated tooth found to be avulsed from its normal position in the bone and located horizontally on the adjacent edentulous alveolar ridge (Figure 2). We ordered a panoramic radiograph to evaluate the current status of the teeth. In the new panoramic view, severe distal root resorption was detected in the migrated tooth which did not exist in the previous radiograph (Figure 3). This type of pathologic tooth migration has not been reported up to now. After routine examinations, the patient was referred to the oral and maxillofacial surgeon, periodontist and prosthodontist for extraction of the migrated tooth and further treatments.

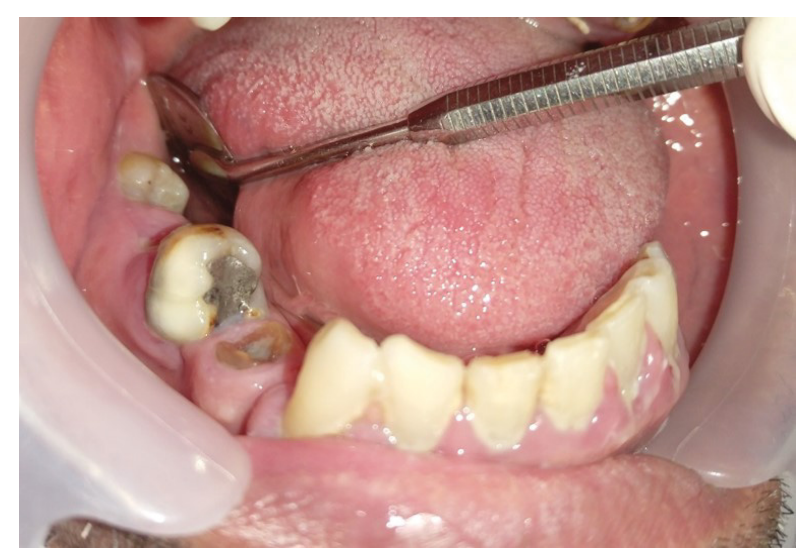

Figure 1: Pathologic tooth migration with an unusual position

*Corresponding author: Fahimeh Anbari, Department of Oral Medicine, Dental School, Shahid Beheshti University of Medical Sciences, Daneshjoo Blvd, Evin Chamran High Way, Tehran, Zip code: 1983963113, Iran, Tel: +98 2122175351; E-mail: fahimeh.anbari@gmail.com

Received May 23, 2018; Accepted November 19, 2018; Published November 22, 2018

Citation: Mortazav H, Rezaeifar K, Anbari F, Rahmani MS (2018) Introducing a New Type of Pathologic Tooth Migration In A Patient with Periodontal Disease. Dentistry 8: 519. doi:10.4172/2161-1122.1000519

Copyright: (c) 2018 Mortazav $\mathrm{H}$, et al. This is an open-access article distributed under the terms of the Creative Commons Attribution License, which permits unrestricted use, distribution, and reproduction in any medium, provided the original author and source are credited. 


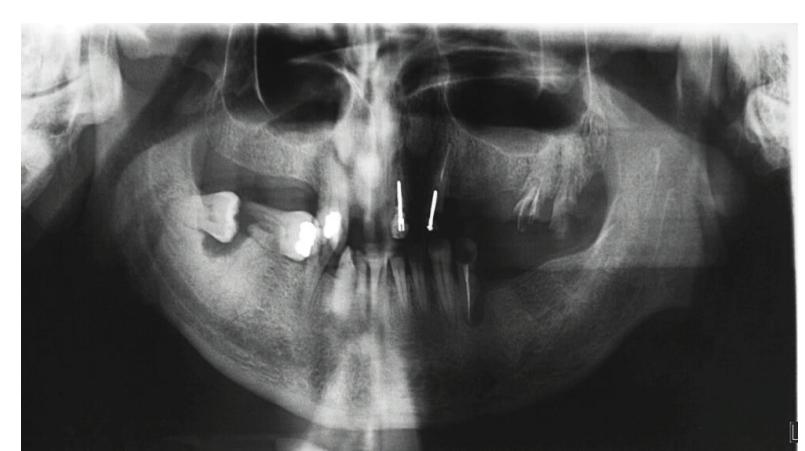

Figure 2: Pathologic tooth migration in a horizontal position, called avulsion.

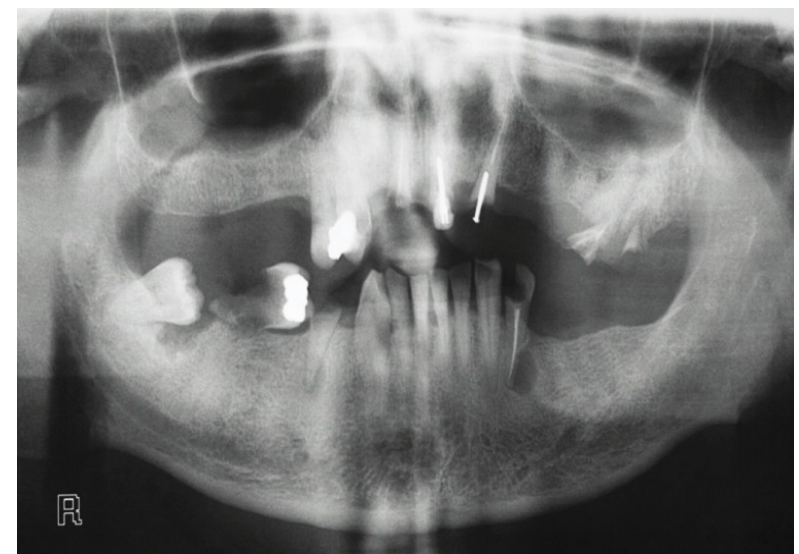

Figure 3: Root resorption of distal root of an avulsed tooth after about two years.

\section{Discussion}

The prevalence of PTM has been reported to be up to $56 \%$ [1]. The mean age of patients with PTM is reported to be from $40.74 \pm$ 11.4 to $46 \pm 11.6$ years according to Khorshidi and Costa, respectively $[1,2]$. There is a sex predilection in PTM with a female to male ratio 2:1 (or 3:1 in some studies) [1,2]. It has been demonstrated that there is a significant relationship between bone loss and this entity [4]. In line with this, Costa evaluate the periodontal conditions of anterior teeth with pathologic migration in patients with chronic periodontitis and showed that patients who presented an average bone loss of $25 \%$ were related to $33.6 \%$ of PTM, while those with bone loss between $50 \%$ and $75 \%$ corresponded to the group with predominance of PTM (80\%) [1]. The degree of Clinical Attachment Loss (CAL) is another risk factor for PTM. According to Towfighi and Rohatgi, the mean of CAL in patients with PTM is expected to be between $3.3 \mathrm{~mm}$ and 5.5 $\mathrm{mm}[5,8]$. This condition is classified into five subgroups clinically and radiographically according to their specific type of movement $[1,4,5]$ :

1-facial flaring; 2-diastema; 3-extrusion; 4-rotation; 5-proximal tilting/tipping/or drifting
In this paper, we reported a new type of PTM which doesn't fit into any of the previous subgroups. We designated it as "avulsion". Therefore, the previous five-category classification of PTM can be modified to a newer one with six subtypes. The affected tooth in our case had been extruded completely and located horizontally on the adjacent alveolar ridge. It was covered with normal mucosa with mild to moderate mobility. This is the most severe type of PTM that has ever been reported. Among the conditions that were mentioned as a possible cause of PTM, periodontitis, missing tooth, posterior tooth loss, bone loss, mouth breathing and protrusive pattern of mastication have been found in our case. In accordance to our patient's condition, Cuervo Mongui demonstrated that patients with obstructive sleep apnea have a greater severity of periodontal disease, fewer remained teeth in the mouth, increased tooth mobility, a greater amount of furcation involvement and a history of less frequent brushing [9]. In addition, Soe found that old age, male gender, current smoking status, mouth breathing during sleep were identified as risk factors for periodontitis. $60.0 \%$ who were diagnosed with periodontitis had sleep apnea. Mouth breathing during sleep was identified as a risk factor for periodontitis in this group of patients [10]. Treatment of most patients with severe PTM requires a multidisciplinary approach of orthodontics, periodontics, and prosthodontics to achieve success. In the early stage of PTM, spontaneous correction of migrated teeth sometimes occurs after periodontal therapy [3]. Fixed orthodontic has been suggested as an important treatment modality in the correction of PTM (4). In conclusion, from now on, PTM can be divided into six subgroups as: 1- facial flaring; 2-diastema; 3- extrusion; 4-rotation; 5-proximal tilting/tipping/or drifting, and 6-avulsion.

\section{References}

1. Costa MR, Silvério KG, Rossa Júnior C, Cirelli JA (2004) Periodontal conditions of teeth presenting pathologic migration. Braz oral res 18: 301-305.

2. Khorshidi H, Moaddeli MR, Golkari A, Heidari H, Raoofi S (2016) The prevalence of pathologic tooth migration with respect to the severity of periodontitis. J Int Soc Prevent Communit Dent 6: 122.

3. Brunsvold MA (2005) Pathologic tooth migration. J Periodontol 76: 859-866

4. Awang RAR, Ismail $\mathrm{NH}$ (2010) Further migration of tooth in a patient with reduced periodontium: A case report. Arch Orofac Sci 5: 65-68.

5. Towfighi PP, Brunsvold MA, Storey AT, Arnold RM, Willman DE (1997) Pathologic migration of anterior teeth in patients with moderate to severe periodontitis. J Periodontol 68: 967-972.

6. Dadlani H, Ramachandra SS, Mehta DS (2013) Spontaneous correction of pathologically migrated teeth with periodontal therapy alone. J Indian Soc Periodontol 17: 531

7. Rangari R, Reddy C (2016) Etiology of pathological tooth migration related to periodontal diseases: A comprehensive review. Int J Curr Res 8: 36362-36364.

8. Rohatgi S, Narula SC, Sharma RK, Tewari S, Bansal P (2011)A study on clinica attachment loss and gingival inflammation as etiologic factors in pathologic tooth migration. Niger J Clin Pract 14: 449-453.

9. Monguí AC, Pastrana MCM, Quiroga GMS, Torres MH, Uriza CL, et al. (2016) Periodontal Status of Patients with Obstructive Sleep Apnea. Univ Odontol 35.

10. Seo WH, Cho ER, Thomas RJ, An SY, Ryu JJ, et al. (2013) The association between periodontitis and obstructive sleep apnea: a preliminary study. J Periodontal Res 48: 500-506. 